\title{
Endoscopic Retrograde Cholangiopancreatography in Recurrent Acute Pancreatitis: Determining the Optimal Subgroup of Patients in Whom the Procedure is Beneficial
}

\author{
Tae Yoon Lee ${ }^{1}$ and Takuji Iwashita ${ }^{2}$ \\ ${ }^{1}$ Division of Gastroenterology, Department of Internal Medicine, Konkuk University School of Medicine, Seoul, Korea, ${ }^{2}$ First Department of \\ Internal Medicine, Gifu University Hospital, Gifu, Japan
}

See "Practice Patterns and Use of Endoscopic Retrograde Cholangiopancreatography in the Management of Recurrent Acute Pancreatitis" by Jonathan B. Reichstein, Vaishali Patel, Parit Mekaroonkamol, et al., on page 73-81.

Recurrent acute pancreatitis (RAP) is a syndrome characterized by relapsing bouts of acute pancreatitis punctuated by asymptomatic or minimally symptomatic intervals of variable durations-ranging from months to years. Patients with RAP are at potential risk of developing chronic pancreatitis over time. Therefore, when managing a patient with RAP, clinicians should make every effort to minimize the likelihood of relapse.

Endoscopic retrograde cholangiopancreatography (ERCP) is generally required as a therapeutic tool after a definite etiology has been identified; meanwhile, tools such as magnetic resonance cholangiopancreatography (MRCP) and endoscopic ultrasound (EUS) are used as less invasive alternatives for diagnosing chronic pancreatitis, ductal abnormalities, and occult obstructive etiologies. However, even among experts there is debate regarding the use of endoscopic treatment for RAP; some advocate the use of ERCP, while others do not. Moreover, the specific interventions performed during ERCP

Received: December 10, 2019 Revised: January 1, 2020

Accepted: January 2, 2020

Correspondence: Takuji Iwashita

First Department of Internal Medicine, Gifu University Hospital, 1-1 Yanagido, Gifu 501-1194, Japan

Tel: +81-58-230-6308, Fax: +81-58-230-6310, E-mail: takuji@w7.dion.ne.jp ORCID: https://orcid.org/0000-0003-4978-1787

(c) This is an Open Access article distributed under the terms of the Creative Commons Attribution Non-Commercial License (http://creativecommons.org/ licenses/by-nc/3.0) which permits unrestricted non-commercial use, distribution, and reproduction in any medium, provided the original work is properly cited. are still heterogenous.

In a study published in this issue of Clinical Endoscopy, Reichstein et al. investigated the clinical patterns of ERCP use in the treatment of RAP through a nationwide survey in the United States. ${ }^{1}$ The study focused on whether or not a patient would undergo ERCP for RAP and did not consider other modalities of evaluation such as EUS or secretin MRCP. They concluded that factors like duct dilatation, presence of symptoms, and pancreas divisum significantly increased the probability that ERCP is recommended for a patient. Of these, pancreatic duct dilatation was found to be the most significant factor, followed by presence of symptoms and pancreas divisum.

Pancreatic duct dilatation can be due to various etiologies such as choledochal cysts, anomalous pancreatobiliary junctions, tumors, papillary stenosis, and sphincter of Oddi dysfunction (SOD). ERCP plays a clear role in patients with RAP secondary to pancreatobiliary neoplasms, type 3 choledochal cysts, and anomalous pancreatobiliary junctions. ${ }^{2} \mathrm{SOD}$ is defined as a pathologic elevation in basal sphincter pressure $(>40$ $\mathrm{mm} \mathrm{Hg}$ ) and might be related to RAP; however, it remains unclear whether SOD represents an underlying cause, or is the result of a fibroinflammatory change around the papilla. ${ }^{2}$ Studies investigating ERCP for SOD based on manometry are generally limited due to a lack of control groups. One longterm study showed that the 10-year recurrence rate for cases of RAP due to SOD treated with ERCP is more than $50 \%{ }^{3}$ 
Therefore, without pancreatic or biliary duct dilatation, the relationship between SOD and RAP is controversial and the benefit of sphincterotomy is doubtful.

Pancreas divisum is generally considered an obstructive etiology of RAP despite substantial controversy pertaining to the treatment of this condition. ERCP has been the standard tool for identifying and managing pancreas divisum. However, most subjects with pancreas divisum do not have pancreatitis. A recent study using MRCP reported that the prevalence of pancreas divisum in patients with RAP is comparable to that in the healthy population ( $8 \%-10 \%)$, but that a higher incidence of genetic abnormalities was found in patients with both pancreas divisum and RAP. ${ }^{4}$ Environmental exposures or morphologic abnormalities like santoriniceles also increase the baseline risk of developing RAP in cases of pancreas divisum, suggesting that pancreas divisum is merely a cofactor rather than a cause. Therefore, the benefit of performing minor papilla sphincterotomy or stenting for all patients with pancreas divisum in RAP is questionable. One randomized study evaluated the impact of such surgical procedures on patients with RAP $(n=19)$, and the results of the follow-up were variable. ${ }^{5}$ Nevertheless, there are compelling retrospective cohort studies suggesting the benefit of surgery ${ }^{6}$; and accordingly, most high-volume ERCP providers offer ERCP for patients with both RAP and pancreas divisum.

There is little data on what kind of sphincterotomy is beneficial for the treatment of RAP. Therefore, this study also surveyed the specific interventions chosen by endoscopists during the index ERCP. In the presence of pancreatic duct dilatation, $66.2 \%$ of the endoscopists chose to perform pancreatic sphincterotomy, $52.3 \%$ chose biliary sphincterotomy, and $32.8 \%$ performed both. A large clinical trial $(n=69)$ comparing patients with RAP and SOD treated with biliary or dual sphincterotomy revealed no difference over time. ${ }^{7}$ At the index ERCP of RAP patients without pancreatic duct dilatation, $45.9 \%$ of endoscopists might perform biliary sphincterotomy. However, the long-term effect of empirical biliary sphincterotomy for the treatment of occult choledocholithiasis or microlithiasis is unproven. While biliary sphincterotomy is safer than pancreatic sphincterotomy, its impact on the clinical course of RAP remains unclear. Without objective evidence to suggest gallstone pancreatitis, biliary sphincterotomy needs to be compared with sham for patients with RAP.
Although there are no guidelines for the role of ERCP in RAP patients, we recommend ERCP in cases of RAP with pancreatobiliary neoplasms, type 3 choledochal cysts, anomalous pancreatobiliary junctions, and SOD with pancreatic or biliary duct dilatation. The choice of ERCP for the treatment of RAP patients with pancreas divisum remains an individualized decision. Given the substantial debate among experts regarding the endoscopic treatment of RAP, this study is a good start to better understanding the treatment of RAP, by evaluating which factors would increase the recommendation of ERCP and which specific interventions are chosen to follow the initial ERCP. We believe that future studies which perform a worldwide survey (beyond the United States) would help to accurately determine the optimal subgroup of patients in whom ERCP may be effective and which interventions are the most beneficial for RAP patients.

\section{Conflicts of Interest}

The authors have no financial conflicts of interest.

ORCID

Tae Yoon Lee: https://orcid.org/0000-0003-1008-9814

\section{REFERENCES}

1. Reichstein JB, Patel V, Mekaroonkamol P, et al. Practice patterns and use of endoscopic retrograde cholangiopancreatography in the management of recurrent acute pancreatitis. Clin Endosc 2020;53:73-81.

2. Guda NM, Muddana V, Whitcomb DC, et al. Recurrent acute pancreatitis: international state-of-the-science conference with recommendations. Pancreas 2018;47:653-666.

3. Wehrmann T. Long-term results ( $\geq 10$ years) of endoscopic therapy for sphincter of Oddi dysfunction in patients with acute recurrent pancreatitis. Endoscopy 2011;43:202-207.

4. Bertin C, Pelletier AL, Vullierme MP, et al. Pancreas divisum is not a cause of pancreatitis by itself but acts as a partner of genetic mutations. Am J Gastroenterol 2012;107:311-317.

5. Lans JI, Geenen JE, Johanson JF, Hogan WJ. Endoscopic therapy in patients with pancreas divisum and acute pancreatitis: a prospective, randomized, controlled clinical trial. Gastrointest Endosc 1992;38:430434.

6. Mariani A, Di Leo M, Petrone MC, et al. Outcome of endotherapy for pancreas divisum in patients with acute recurrent pancreatitis. World J Gastroenterol 2014;20:17468-17475

7. Coté GA, Imperiale TF, Schmidt SE, et al. Similar efficacies of biliary, with or without pancreatic, sphincterotomy in treatment of idiopathic recurrent acute pancreatitis. Gastroenterology 2012;143:1502-1509.e1. 